To appear in Statistics: A Journal of Theoretical and Applied Statistics

Vol. 00, No. 00, Month 20XX, 1-15

\title{
Martingale inequalities of type Dzhaparidze and van Zanten
}

\author{
X. Fan ${ }^{\mathrm{a} *}$, I. Grama ${ }^{\mathrm{b}}$ and Q. Liu ${ }^{\mathrm{b}}$ \\ ${ }^{a}$ Regularity Team, Inria, France; ${ }^{b}$ Université de Bretagne-Sud, UMR 6205, LMBA, Vannes, \\ France.
}

(v5.0 released February 2015)

\begin{abstract}
Freedman's inequality is a supermartingale counterpart to Bennett's inequality. This result shows that the tail probabilities of a supermartingale is controlled by the quadratic characteristic and a uniform upper bound for the supermartingale difference sequence. Replacing the quadratic characteristic by $\mathrm{H}_{k}^{y}:=\sum_{i=1}^{k}\left(\mathbf{E}\left(\xi_{i}^{2} \mid \mathcal{F}_{i-1}\right)+\xi_{i}^{2} \mathbf{1}_{\left\{\left|\xi_{i}\right|>y\right\}}\right)$, Dzhaparidze and van Zanten (Stochastic Process. Appl., 2001) have extended Freedman's inequality to martingales with unbounded differences. In this paper, we prove that $\mathrm{H}_{k}^{y}$ can be refined to $\mathrm{G}_{k}^{y}:=\sum_{i=1}^{k}\left(\mathbf{E}\left(\xi_{i}^{2} \mathbf{1}_{\left\{\xi_{i} \leq y\right\}} \mid \mathcal{F}_{i-1}\right)+\xi_{i}^{2} \mathbf{1}_{\left\{\xi_{i}>y\right\}}\right)$. Moreover, we also establish two inequalities of type Dzhaparidze and van Zanten. These results extend Sason's inequality (Statist. Probab. Lett., 2012) to the martingales with possibly unbounded differences and establish the connection between Sason's inequality and De la Peña's inequality (Ann. Probab., 1999). An application to self-normalized deviations is given.
\end{abstract}

Keywords: Freedman's inequality; De la Peña's inequality; exponential inequalities; tail probabilities; martingales; self-normalized deviations

AMS Subject Classification: 60G42; 60E15

\section{Introduction}

Exponential inequalities for tail probabilities of sums of independent real-valued random variables and their extension to martingales have numerous important applications in probability theory and statistics. See, for instance, De la Peña and Pang [4], Bercu and Touati [2] and [8]. The classical Bennett inequality [1] gives a tail bound for sums of independent random variables with a bounded range. If $\left(\xi_{i}\right)_{i \geq 1}$ are zero-mean independent random variables, all bounded by some constant $a$ so that $\left|\xi_{i}\right| \leq a$ for all $i$, then the sum $S_{n}=\sum_{i=1}^{n} \xi_{i}$ obeys the following Bennett inequality (see also Bernstein [3]): for all $x>0$,

$$
\begin{aligned}
\mathbf{P}\left(S_{n} \geq x\right) & \leq B_{1}(x, a, v):=\left(\frac{v^{2}}{x a+v^{2}}\right)^{\frac{x}{a}+\frac{v^{2}}{a^{2}}} e^{\frac{x}{a}} \\
& \leq B_{2}(x, a, v):=\exp \left\{-\frac{x^{2}}{2\left(v^{2}+x a / 3\right)}\right\}
\end{aligned}
$$

where $v^{2}$ is the variance of $S_{n}$.

*Corresponding author. Email: fanxiequan@hotmail.com 
Freedman have extended Bennett's result to the case of discrete-time supermartingales with bounded jumps. Let $\left(\xi_{i}, \mathcal{F}_{i}\right)_{i=1, \ldots, n}$ be a sequence of supermartingale differences. Denote by $S_{k}=\sum_{i=1}^{k} \xi_{i}$ and $\langle S\rangle_{k}=\sum_{i=1}^{k} \mathbf{E}\left(\xi_{i}^{2} \mid \mathcal{F}_{i-1}\right)$. The well-known Freedman's inequality [9] for supermartingales states that: if $\xi_{i} \leq a$ for a positive constant $a$, then, for all $x, v>0$,

$$
\begin{aligned}
\mathbf{P}\left(S_{k} \geq x \text { and }\langle S\rangle_{k} \leq v^{2} \text { for some } k \in[1, n]\right) & \leq B_{1}(x, a, v) \\
& \leq B_{2}(x, a, v)
\end{aligned}
$$

In particular, when $\left(\xi_{i}\right)_{i=1, \ldots, n}$ are independent, the bounds (3) and (4) reduce to the bounds of Bennett [1] and Bernstein [3], respectively. Moreover, Freedman's inequality implies that the bounds (3) and (4) not only hold for $S_{n}$ but even hold for the maximum of partial sums $\max _{1 \leq k \leq n} S_{k}$.

Replacing the quadratic characteristic $\langle S\rangle_{k}$ by

$$
\mathrm{H}_{k}^{y}:=\sum_{i=1}^{k}\left(\mathbf{E}\left(\xi_{i}^{2} \mid \mathcal{F}_{i-1}\right)+\xi_{i}^{2} \mathbf{1}_{\left\{\left|\xi_{i}\right|>y\right\}}\right),
$$

Dzhaparidze and van Zanten [7] have established a generalization of Freedman's inequality for martingales with unbounded differences: for all $x, y, v>0$,

$$
\mathbf{P}\left(S_{k} \geq x \text { and } \mathrm{H}_{k}^{y} \leq v^{2} \text { for some } k \in[1, n]\right) \leq B_{1}(x, y, v) .
$$

In particular, if $\left|\xi_{i}\right| \leq a$ for all $i$, it holds $\mathrm{H}_{k}^{a}=\langle S\rangle_{k}$, and then the inequality of Dzhaparidze and van Zanten (5) reduces to Freeman's inequality (3), implying inequality (4).

However, if $\left(\xi_{i}\right)$ are not bounded from below, inequality (5) does not imply Freeman's inequality (3) in general. To fill this gap, we propose replacing the random variable $\mathrm{H}_{k}^{y}$ in inequality (5) by a smaller one $\mathrm{G}_{k}^{y}$, where

$$
\mathrm{G}_{k}^{y}=\sum_{i=1}^{k}\left(\mathbf{E}\left(\xi_{i}^{2} \mathbf{1}_{\left\{\xi_{i} \leq y\right\}} \mid \mathcal{F}_{i-1}\right)+\xi_{i}^{2} \mathbf{1}_{\left\{\xi_{i}>y\right\}}\right) .
$$

Our Theorem 2.1 states that, for all $x, y \geq 0$ and $v>0$,

$$
\mathbf{P}\left(S_{k} \geq x \text { and } \mathrm{G}_{k}^{y} \leq v^{2} \text { for some } k \in[1, n]\right) \leq B_{1}(x, y, v) .
$$

Since $\mathrm{G}_{k}^{y} \leq \mathrm{H}_{k}^{y}$, inequality (7) implies the inequality of Dzhaparidze and van Zanten (5). Moreover, if $\xi_{i} \leq a$ for all $i \in[1, n]$ (may not be bounded from below), it holds $\mathrm{G}_{k}^{a}=\langle S\rangle_{k}$ for all $k \in[1, n]$, and then (7) also implies Freeman's inequality (3). This fills the gap.

In Theorem [2.2, we give a generalization of (5) to the supermartingales with nonsquare-integrable differences. Write

$$
\mathrm{G}_{n}(\beta)=\sum_{i=1}^{n}\left(\mathbf{E}\left(\left|\xi_{i}\right|^{\beta} \mid \mathcal{F}_{i-1}\right)+\left|\xi_{i}\right|^{\beta}\right)
$$


for a constant $\beta \in(1,2)$. Then, for all $x, v>0$,

$$
\mathbf{P}\left(\max _{1 \leq k \leq n} S_{k} \geq x \text { and } \mathrm{G}_{n}(\beta) \leq v^{\beta}\right) \leq \exp \left\{-C(\beta)\left(\frac{x}{v}\right)^{\frac{\beta}{\beta-1}}\right\}
$$

where $C(\beta)=\beta^{\frac{1}{1-\beta}}\left(1-\beta^{-1}\right)$.

Under the additional assumption of conditional symmetry, Sason [12] gave an improvements of Freedman's inequality (4). Sason proved that if $\left(\xi_{i}, \mathcal{F}_{i}\right)_{i \geq 1}$ is a sequence of conditionally symmetric martingale differences with $\left|\xi_{i}\right| \leq a$ for a positive constant $a$, then, for all $x, v>0$,

$$
\begin{aligned}
& \mathbf{P}\left(S_{k} \geq x \text { and }\langle S\rangle_{k} \leq v^{2} \text { for some } k \in[1, n]\right) \\
& \quad \leq B_{0}(x, a, v):=\exp \left\{-\lambda x+\left(\frac{\cosh (\lambda a)-1}{a^{2}}\right) v^{2}\right\}
\end{aligned}
$$

and $B_{0}(x, a, v) \leq B_{1}(x, a, v)$, where

$$
\lambda=\frac{1}{a} \log \left(\sqrt{1+\frac{x^{2} a^{2}}{v^{4}}}+\frac{x a}{v^{2}}\right) .
$$

In the spirit of Dzhaparide and van Zanten [7], we establish the following generalization of Sason's inequality (9). Define

$$
\mathrm{M}_{k}^{y}=\sum_{i=1}^{k}\left(\mathbf{E}\left(\xi_{i}^{2} \mathbf{1}_{\left\{\left|\xi_{i}\right| \leq y\right\}} \mid \mathcal{F}_{i-1}\right)+\xi_{i}^{2} \mathbf{1}_{\left\{\left|\xi_{i}\right|>y\right\}}\right) .
$$

Then, for all $x, v>0$ and all $y \geq 0$,

$$
\mathbf{P}\left(S_{k} \geq x \text { and } \mathrm{M}_{k}^{y} \leq v^{2} \text { for some } k \in[1, n]\right) \leq B_{0}(x, y, v),
$$

where, by convention,

$$
B_{0}(x, 0, v)=\lim _{y \rightarrow 0+} B_{0}(x, y, v)=\exp \left\{-\frac{x^{2}}{2 v^{2}}\right\}
$$

applied when $y=0$. If $\left|\xi_{i}\right| \leq a$ for all $i$, then $\mathrm{M}_{k}^{a}=\langle S\rangle_{k}$ for all $k$ and (11) reduces to Sason's inequality (9). Notice that when $y=0$, inequality (11) is known as De la Peña's inequality [5]. Hence, our bound establishes a connection between the inequalities of De la Peña and Sason.

The paper is organized as follows. We present our main results in Section 2 and the application to self-normalized deviations in Section [3, and devote to the proofs of the main results in Sections $4-6$.

\section{Main results}

Assume that we are given a sequence of real-valued supermartingale differences $\left(\xi_{i}, \mathcal{F}_{i}\right)_{i=0, \ldots, n}$ defined on some probability space $(\Omega, \mathcal{F}, \mathbf{P})$, where $\xi_{0}=0$ and $\{\emptyset, \Omega\}=$ 
$\mathcal{F}_{0} \subseteq \ldots \subseteq \mathcal{F}_{n} \subseteq \mathcal{F}$ are increasing $\sigma$-fields. So we have $\mathbf{E}\left(\xi_{i} \mid \mathcal{F}_{i-1}\right) \leq 0, i=1, \ldots, n$, by definition. Set

$$
S_{k}=\sum_{i=1}^{k} \xi_{i}, \quad k=1, \ldots, n
$$

Then $S=\left(S_{k}, \mathcal{F}_{k}\right)_{k=1, \ldots, n}$ is a supermartingale. Let $\langle S\rangle$ be the quadratic characteristic and $[S]$ be the squared variation of the supermartingale $S$ :

$$
\langle S\rangle_{k}=\sum_{i=1}^{k} \mathbf{E}\left(\xi_{i}^{2} \mid \mathcal{F}_{i-1}\right) \quad \text { and } \quad[S]_{k}=\sum_{i=1}^{k} \xi_{i}^{2}
$$

The following theorem strengthens the inequality of Dzhaparide and van Zanten [7].

Theorem 2.1 Assume $\mathbf{E} \xi_{i}^{2}<\infty$ for all $i \in[1, n]$. Then, for all $x, y \geq 0$ and $v>0$,

$$
\begin{aligned}
\mathbf{P}\left(S_{k} \geq x \text { and } G_{k}^{y} \leq v^{2} \text { for some } k \in[1, n]\right) & \leq B_{1}(x, y, v) \\
& \leq B_{2}(x, y, v) .
\end{aligned}
$$

where $G_{k}^{y}$ is defined by (6) and

$$
B_{1}(x, 0, v)=\lim _{y \rightarrow 0+} B_{1}(x, y, v)=B_{2}(x, 0, v)
$$

applied when $y=0$.

Since $\mathrm{G}_{k}^{0} \leq\langle S\rangle_{n}+[S]_{n}$ for all $k \in[1, n]$, inequality (15) implies the following result: for all $x, v>0$,

$$
\mathbf{P}\left(\max _{1 \leq k \leq n} S_{k} \geq x \text { and }\langle S\rangle_{n}+[S]_{n} \leq v^{2}\right) \leq B_{1}(x, 0, v)
$$

This result slightly refines an earlier inequality of Bercu and Touati [2], where they have obtained the same bound on tail probabilities $\mathbf{P}\left(S_{n} \geq x\right.$ and $\left.\langle S\rangle_{n}+[S]_{n} \leq v^{2}\right)$. Thus the sum $S_{n}$ has been strengthened to the maximum of partial sums $\max _{1 \leq k \leq n} S_{k}$. A similar refinement is applied to Delyon's inequality [6], where he has established the following result, for all $x, v>0$,

$$
\mathbf{P}\left(S_{n} \geq x \text { and } \mathrm{G}_{n}^{0} \leq v^{2}\right) \leq B_{2}(x, 0, v) .
$$

Consider the supermartingales with non-square-integrable differences. We have the following large deviation exponential bound, which can be regarded as a generalization of Delyon's inequality (17) or the inequality of Dzhaparide and van Zanten (5).

Denote by $x^{+}=\max \{x, 0\}$ and $x^{-}=-\min \{x, 0\}$ the positive and negative parts of $x$, respectively.

Theorem 2.2 Assume $\mathbf{E}\left|\xi_{i}\right|^{\beta}<\infty$ for a constant $\beta \in(1,2)$ and all $i \in[1, n]$. Write

$$
G_{k}^{0}(\beta)=\sum_{i=1}^{k}\left(\mathbf{E}\left(\left(\xi_{i}^{-}\right)^{\beta} \mid \mathcal{F}_{i-1}\right)+\left(\xi_{i}^{+}\right)^{\beta}\right), \quad k \in[1, n] .
$$


Then, for all $x, v>0$,

$$
\mathbf{P}\left(S_{k} \geq x \text { and } G_{k}^{0}(\beta) \leq v^{\beta} \text { for some } k \in[1, n]\right) \leq \exp \left\{-C(\beta)\left(\frac{x}{v}\right)^{\frac{\beta}{\beta-1}}\right\}
$$

where

$$
C(\beta)=\beta^{\frac{1}{1-\beta}}\left(1-\beta^{-1}\right) .
$$

In particular, if $\left\|\mathrm{G}_{n}(\beta)\right\|_{\infty}=O(n)$ as $n \rightarrow \infty$, then, for any $x>0$,

$$
\mathbf{P}\left(\max _{1 \leq k \leq n} S_{k} \geq n x\right)=O\left(\exp \left\{-n C_{x}(\beta)\right\}\right)
$$

where $C_{x}(\beta)>0$ does not depend on $n$.

It is also interesting to see that when $\beta$ decreases to 1 in (18), the power $\frac{\beta}{\beta-1}$ is increasing to infinity and the corresponding constant $C(\beta)$ is decreasing to 0 . This means the larger the power, the smaller the corresponding constant.

One calls $\left(\xi_{i}, \mathcal{F}_{i}\right)_{i \geq 1}$ a sequence of conditionally symmetric martingale differences, if $\mathbf{E}\left(\xi_{i}>y \mid \mathcal{F}_{i-1}\right)=\mathbf{E}\left(\xi_{i}<-y \mid \mathcal{F}_{i-1}\right)$ for all $i$ and any $y \geq 0$. Motivated by the result of Dzhaparide and van Zanten [7], we give a generalization of Sason's inequality (9) to the martingales with unbounded differences.

THEOREM 2.3 Assume that $\left(\xi_{i}, \mathcal{F}_{i}\right)_{i \geq 1}$ is a sequence of conditionally symmetric martingale differences. Then, for all $x, v>0$ and all $y \geq 0$,

$$
\mathbf{P}\left(S_{k} \geq x \text { and } M_{k}^{y} \leq v^{2} \text { for some } k \in[1, n]\right) \leq B_{0}(x, y, v),
$$

where $M_{k}^{y}$ is defined by (10).

If the martingale differences are bounded $\left|\xi_{i}\right| \leq a$ for a positive constant $a$, then $\mathrm{M}_{k}^{a}=\langle S\rangle_{k}$ and inequality (21) with $y=a$ reduces to Sason's inequality (9). As pointed out by Sason [12], inequality (21) is the best possible that can be obtained from Chernoff's inequality $\mathbf{P}\left(S_{n} \geq x\right) \leq \inf _{\lambda \geq 0} \mathbf{E} e^{\lambda\left(S_{n}-x\right)}$ under the present assumption in a certain sense. Indeed, if $\left(\xi_{i}\right)_{i \geq 1}$ are i.i.d. random variables and satisfy the following distribution

$$
\mathbf{P}\left(\xi_{i}=y\right)=\mathbf{P}\left(\xi_{i}=-y\right)=\frac{v^{2}}{2 n y^{2}} \quad \text { and } \quad \mathbf{P}\left(\xi_{i}=0\right)=1-\frac{v^{2}}{n y^{2}},
$$

then the bound (21) equals to $\lim _{n \rightarrow \infty} \inf _{\lambda \geq 0} \mathbf{E} e^{\lambda\left(S_{n}-x\right)}$.

Since $\lim _{y \rightarrow 0} \lambda=\frac{x}{v^{2}}$ and $\mathrm{G}_{k}^{0}=[S]_{k}$, inequality (21) reduces to De la Peña's inequality [5] as $y \rightarrow 0$ : for all $x, v>0$,

$$
\mathbf{P}\left(S_{k} \geq x \text { and }[S]_{k} \leq v^{2} \text { for some } k \in \mathbb{N}\right) \leq B_{0}(x, 0, v) .
$$

Thus inequality (21) establishes the connection between the inequalities of De la Peña and Sason. 


\section{Application to self-normalized deviations}

As an application of Theorem 2.2 , consider the self-normalized deviations for independent random variables.

THEOREM 3.1 Assume that $\left(\xi_{i}\right)_{i=1, \ldots, n}$ is a sequence of independent and symmetric random variables. Denote by

$$
V_{n}(\beta)=\left(\sum_{i=1}^{n}\left|\xi_{i}\right|^{\beta}\right)^{1 / \beta}
$$

for a constant $\beta \in(1,2]$. Then, for all $x>0$,

$$
\mathbf{P}\left(\max _{1 \leq k \leq n} S_{k} / V_{n}(\beta) \geq x\right) \leq \exp \left\{-\widetilde{C}(\beta) x^{\frac{\beta}{\beta-1}}\right\}
$$

where

$$
\widetilde{C}(\beta)=\left(\frac{\beta}{2}\right)^{\frac{1}{1-\beta}}\left(1-\beta^{-1}\right)
$$

Proof of Theorem 3.1. Assume that $\left(\xi_{i}\right)_{i=1, \ldots, n}$ are independent and symmetric. Set

$$
\mathcal{F}_{i}=\sigma\left\{\xi_{k}, k \leq i,\left|\xi_{j}\right|, 1 \leq j \leq n\right\} .
$$

Since $\xi_{i}$ is symmetric, it is easy to see that

$$
\mathbf{E}\left(\frac{\xi_{i}}{\mathrm{~V}_{n}(\beta)} \mid \mathcal{F}_{i-1}\right)=\mathbf{E}\left(\xi_{i}|| \xi_{i} \mid\right) \frac{1}{\mathrm{~V}_{n}(\beta)}=0
$$

Therefore, $\left(\xi_{i} / \mathrm{V}_{n}(\beta), \mathcal{F}_{i}\right)_{i=1, \ldots, n}$ is a sequence of martingale differences. Notice that

$$
\begin{aligned}
\frac{1}{\mathrm{~V}_{n}(\beta)^{\beta}} \sum_{i=1}^{k}\left(\mathbf{E}\left(\left(\xi_{i}^{-}\right)^{\beta} \mid \mathcal{F}_{i-1}\right)+\left(\xi_{i}^{+}\right)^{\beta}\right) & \leq \frac{1}{\mathrm{~V}_{n}(\beta)^{\beta}} \sum_{i=1}^{n}\left(\mathbf{E}\left(\left|\xi_{i}\right|^{\beta} \mid \mathcal{F}_{i-1}\right)+\left|\xi_{i}\right|^{\beta}\right) \\
& =\frac{2}{\mathrm{~V}_{n}(\beta)^{\beta}} \sum_{i=1}^{n}\left|\xi_{i}\right|^{\beta}=2 .
\end{aligned}
$$

Applying Theorem 2.2 to $\left(\xi_{i} / \mathrm{V}_{n}(\beta), \mathcal{F}_{i}\right)_{i=1, \ldots, n}$, we obtain (24).

The power $x^{\frac{\beta}{\beta-1}}$ in (24) is the best possible for $x$ in the moderate deviation and large deviation ranges. Indeed, Jing, Liang and Zhou [10] have obtained the following selfnormalized moderate deviation result. Assume that

$$
\mathbf{P}\left(\xi_{i} \geq x\right)=\mathbf{P}\left(\xi_{i} \leq-x\right) \sim \frac{c}{x^{\alpha}} h_{i}(x), \quad x \rightarrow \infty,
$$

where $\alpha \in(0,2), c>0$ and $h_{i}(x)$ 's are slowly varying at $\infty$. Under certain conditions on the tail probabilities of $\xi_{i}$ (cf. Theorem 2.3 of [10] for details), for $x_{n} \rightarrow \infty$ and 
$x_{n}=o\left(n^{(\beta-1) / \beta}\right)$ and $\beta>\max \{1, \alpha\}$, the limit exists

$$
\lim _{n \rightarrow \infty} x_{n}^{-\frac{\beta}{\beta-1}} \log \mathbf{P}\left(S_{n} / \mathrm{V}_{n}(\beta) \geq x_{n}\right)=-(\beta-1) C_{\alpha}(\beta),
$$

where $C_{\alpha}(\beta)$ is a positive constant depending on $\alpha$ and $\beta$. Equality (26) suggests that the power $x^{\frac{\beta}{\beta-1}}$ in (24) is the best possible for moderate $x$ 's. See also Shao [13] for self-normalized large deviation result.

\section{Proof of Theorem 2.1}

Assume $\left(\xi_{i}, \mathcal{F}_{i}\right)_{i=0, \ldots, n}$ a sequence of square integrable supermartingale differences. For any nonnegative numbers $y$ and $\lambda$, define the exponential multiplicative martingale $Z(\lambda)=\left(Z_{k}(\lambda), \mathcal{F}_{k}\right)_{k=0, \ldots, n}$, where

$$
Z_{k}(\lambda)=\prod_{i=1}^{k} \frac{\exp \left\{\lambda \xi_{i}-\frac{1}{2}\left(\lambda \xi_{i}\right)^{2} \mathbf{1}_{\left\{\xi_{i}>y\right\}}\right\}}{\mathbf{E}\left(\exp \left\{\lambda \xi_{i}-\frac{1}{2}\left(\lambda \xi_{i}\right)^{2} \mathbf{1}_{\left\{\xi_{i}>y\right\}}\right\} \mid \mathcal{F}_{i-1}\right)}, \quad Z_{0}(\lambda)=1
$$

If $T$ is a stopping time, then $Z_{T \wedge k}(\lambda), \lambda>0$, is also a martingale, where

$$
Z_{T \wedge k}(\lambda)=\prod_{i=1}^{T \wedge k} \frac{\exp \left\{\lambda \xi_{i}-\frac{1}{2}\left(\lambda \xi_{i}\right)^{2} \mathbf{1}_{\left\{\xi_{i}>y\right\}}\right\}}{\mathbf{E}\left(\exp \left\{\lambda \xi_{i}-\frac{1}{2}\left(\lambda \xi_{i}\right)^{2} \mathbf{1}_{\left\{\xi_{i}>y\right\}}\right\} \mid \mathcal{F}_{i-1}\right)}, \quad Z_{0}(\lambda)=1 .
$$

Then for any nonnegative number $\lambda$, we have the following conjugate probability measure $\mathbf{P}_{\lambda}$ on $(\Omega, \mathcal{F})$ :

$$
d \mathbf{P}_{\lambda}=Z_{T \wedge n}(\lambda) d \mathbf{P}
$$

LEMma 4.1 For all $y \geq 0$ and all $\lambda>0$, it holds

$\mathbf{E}\left(\exp \left\{\lambda \xi_{i}-\frac{1}{2}\left(\lambda \xi_{i}\right)^{2} \mathbf{1}_{\left\{\xi_{i}>y\right\}}\right\} \mid \mathcal{F}_{i-1}\right) \leq \exp \left\{\left(\frac{e^{\lambda y}-1-\lambda y}{y^{2}}\right) \mathbf{E}\left(\xi_{i}^{2} \mathbf{1}_{\left\{\xi_{i} \leq y\right\}} \mid \mathcal{F}_{i-1}\right)\right\}$

where, by convention, $\frac{e^{\lambda y}-1-\lambda y}{y^{2}}=\frac{\lambda^{2}}{2}$ applied when $y=0$.

Proof. Let $y \geq 0$. If $\xi_{i} \leq y$, since the function

$$
g(x)=\frac{e^{x}-1-x}{x^{2}}
$$

is increasing in $x \in \mathbf{R}$ (by convention $g(0)=1 / 2$ ), we have, for all $\lambda>0$,

$$
\frac{e^{\lambda \xi_{i}}-1-\lambda \xi_{i}}{\left(\lambda \xi_{i}\right)^{2}} \leq \frac{e^{\lambda y}-1-\lambda y}{(\lambda y)^{2}}
$$


If $\xi_{i}>y$, since $\exp \left\{x-\frac{1}{2} x^{2}\right\} \leq 1+x$ for all $x \geq 0$, it follows that, for all $\lambda>0$,

$$
\exp \left\{\lambda \xi_{i}-\frac{1}{2}\left(\lambda \xi_{i}\right)^{2}\right\} \leq 1+\lambda \xi_{i}
$$

Combining (28) and (29) together, we find that, for all $y \geq 0$ and all $\lambda>0$,

$$
\exp \left\{\lambda \xi_{i}-\frac{1}{2}\left(\lambda \xi_{i}\right)^{2} \mathbf{1}_{\left\{\xi_{i}>y\right\}}\right\} \leq 1+\lambda \xi_{i}+\left(\frac{e^{\lambda y}-1-\lambda y}{y^{2}}\right) \xi_{i}^{2} \mathbf{1}_{\left\{\xi_{i} \leq y\right\}} .
$$

Taking conditional expectations on both sides of the last inequality, we deduce that

$$
\mathbf{E}\left(\exp \left\{\lambda \xi_{i}-\frac{1}{2}\left(\lambda \xi_{i}\right)^{2} \mathbf{1}_{\left\{\xi_{i}>y\right\}}\right\} \mid \mathcal{F}_{i-1}\right) \leq 1+\left(\frac{e^{\lambda y}-1-\lambda y}{y^{2}}\right) \mathbf{E}\left(\xi_{i}^{2} \mathbf{1}_{\left\{\xi_{i} \leq y\right\}} \mid \mathcal{F}_{i-1}\right)
$$

Using the inequality $1+x \leq e^{x}$ in the right-hand side of (30), we obtain the desired inequality.

Proof of Theorem 2.1. For any $x, v>0$ and any $y \geq 0$, define the stopping time $T$ :

$$
T(x, y, v)=\min \left\{k \in[1, n]: S_{k} \geq x \text { and } G_{k}^{y} \leq v^{2}\right\}
$$

with the convention that $\min \emptyset=0$. Then it follows that

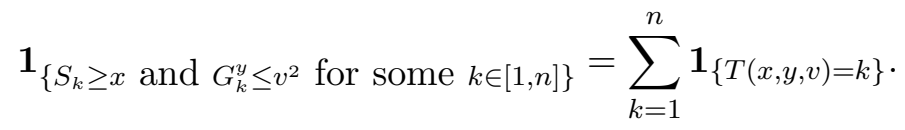

Denote by $\mathbf{E}_{\lambda}$ the expectation with respect to the conjugate probability measure $\mathbf{P}_{\lambda}$. Using the change of probability measure (27), we have, for all $x, \lambda, v>0$ and all $y \geq 0$,

$$
\begin{aligned}
& \mathbf{P}\left(S_{k} \geq x \text { and } G_{k}^{y} \leq v^{2} \text { for some } k \in[1, n]\right) \\
= & \left.\mathbf{E}_{\lambda}\left(Z_{T \wedge n}(\lambda)^{-1} \mathbf{1}_{\left\{S_{k} \geq x\right.} \text { and } G_{k}^{y} \leq v^{2} \text { for some } k \in[1, n]\right\}\right) \\
= & \sum_{k=1}^{n} \mathbf{E}_{\lambda}\left(\exp \left\{-\lambda S_{k}+\frac{\lambda^{2}}{2}[S]_{k}(y)+\widetilde{\Psi}_{k}(\lambda)\right\} \mathbf{1}_{\{T(x, y, v)=k\}}\right),
\end{aligned}
$$

where

$$
[S]_{k}(y)=\sum_{i=1}^{k} \xi_{i}^{2} \mathbf{1}_{\left\{\xi_{i}>y\right\}}
$$

and

$$
\widetilde{\Psi}_{k}(\lambda)=\sum_{i=1}^{k} \log \mathbf{E}\left(\exp \left\{\lambda \xi_{i}-\frac{1}{2}\left(\lambda \xi_{i}\right)^{2} \mathbf{1}_{\left\{\xi_{i}>y\right\}}\right\} \mid \mathcal{F}_{i-1}\right)
$$


Since the function $g(x)$ in increasing in $x$ and $g(0)=1 / 2$, we have

$$
\frac{\lambda^{2}}{2} \leq \frac{e^{\lambda y}-1-\lambda y}{y^{2}} \quad \text { for all } y, \lambda>0
$$

Hence, by Lemma 4.1 and the last inequality,

$$
\begin{aligned}
& \mathbf{P}\left(S_{k} \geq x \text { and } G_{k}^{y} \leq v^{2} \text { for some } k \in[1, n]\right) \\
\leq & \sum_{k=1}^{n} \mathbf{E}_{\lambda}\left(\exp \left\{-\lambda S_{k}+\frac{\lambda^{2}}{2}[S]_{k}(y)+\left(\frac{e^{\lambda y}-1-\lambda y}{y^{2}}\right)\langle S\rangle_{k}(y)\right\} \mathbf{1}_{\{T(x, y, v)=k\}}\right) \\
\leq & \sum_{k=1}^{n} \mathbf{E}_{\lambda}\left(\exp \left\{-\lambda S_{k}+\left(\frac{e^{\lambda y}-1-\lambda y}{y^{2}}\right) G_{k}^{y}\right\} \mathbf{1}_{\{T(x, y, v)=k\}}\right)
\end{aligned}
$$

where $\langle S\rangle_{k}(y)=\sum_{i=1}^{k} \mathbf{E}\left(\xi_{i}^{2} \mathbf{1}_{\left\{\xi_{i} \leq y\right\}} \mid \mathcal{F}_{i-1}\right)$. Therefore, by the fact $S_{k} \geq x$ and $G_{k}^{y} \leq v^{2}$ on the set $\{T(x, y, v)=k\}$, inequality (32) implies that, for all $x, \lambda, v>0$ and all $y \geq 0$,

$$
\begin{aligned}
& \mathbf{P}\left(S_{k} \geq x \text { and } G_{k}^{y} \leq v^{2} \text { for some } k \in[1, n]\right) \\
\leq & \sum_{k=1}^{n} \mathbf{E}_{\lambda}\left(\exp \left\{-\lambda x+\left(\frac{e^{\lambda y}-1-\lambda y}{y^{2}}\right) v^{2}\right\} \mathbf{1}_{\{T(x, y, v)=k\}}\right) \\
\leq & \exp \left\{-\lambda x+\left(\frac{e^{\lambda y}-1-\lambda y}{y^{2}}\right) v^{2}\right\} .
\end{aligned}
$$

The last inequality attains its minimum at

$$
\lambda=\lambda(x)=\frac{1}{y} \log \left(1+\frac{x y}{v^{2}}\right) .
$$

Substituting $\lambda=\lambda(x)$ in (33), we obtain (14). Using the inequality

$$
e^{t}-1-t \leq \frac{t^{2}}{2\left(1-\frac{t}{3}\right)}, \quad t \geq 0
$$

we get, for all $x, v>0$ and all $y \geq 0$,

$$
\begin{aligned}
\inf _{\lambda>0} \exp \left\{-\lambda x+\left(\frac{e^{\lambda y}-1-\lambda y}{y^{2}}\right) v^{2}\right\} & \leq \inf _{\lambda>0} \exp \left\{-\lambda x+\frac{\lambda^{2} v^{2}}{2\left(1-\frac{\lambda y}{3}\right)}\right\} \\
& \leq B_{2}(x, y, v) .
\end{aligned}
$$

Thus (14) implies (15). This completes the proof of Theorem 2.1.

\section{Proof of Theorem 2.2}

Assume $\mathbf{E}\left|\xi_{i}\right|^{\beta}<\infty$ for a constant $\beta \in(1,2)$ and for all $i \in[1, n]$. For any nonnegative numbers $\lambda$, define the exponential multiplicative martingale $Z(\lambda)=\left(Z_{k}(\lambda), \mathcal{F}_{k}\right)_{k=0, \ldots, n}$, 
where

$$
Z_{k}(\lambda)=\prod_{i=1}^{k} \frac{\exp \left\{\lambda \xi_{i}-\left(\lambda \xi_{i}^{+}\right)^{\beta}\right\}}{\mathbf{E}\left(\exp \left\{\lambda \xi_{i}-\left(\lambda \xi_{i}^{+}\right)^{\beta}\right\} \mid \mathcal{F}_{i-1}\right)}, \quad Z_{0}(\lambda)=1
$$

If $T$ is a stopping time, then $Z_{T \wedge k}(\lambda), \lambda \geq 0$, is also a martingale, where

$$
Z_{T \wedge k}(\lambda)=\prod_{i=1}^{T \wedge k} \frac{\exp \left\{\lambda \xi_{i}-\left(\lambda \xi_{i}^{+}\right)^{\beta}\right\}}{\mathbf{E}\left(\exp \left\{\lambda \xi_{i}-\left(\lambda \xi_{i}^{+}\right)^{\beta}\right\} \mid \mathcal{F}_{i-1}\right)}, \quad Z_{0}(\lambda)=1 .
$$

Then for any nonnegative number $\lambda$, we introduce the following conjugate probability measure $\mathbf{P}_{\lambda}$ on $(\Omega, \mathcal{F})$ :

$$
d \mathbf{P}_{\lambda}=Z_{T \wedge n}(\lambda) d \mathbf{P}
$$

Lemma 5.1 If $\mathbf{E}\left|\xi_{i}\right|^{\beta}<\infty$ for a constant $\beta \in(1,2)$, then, for all $\lambda>0$,

$$
\mathbf{E}\left(\exp \left\{\lambda \xi_{i}-\lambda^{\beta}\left(\xi_{i}^{+}\right)^{\beta}\right\} \mid \mathcal{F}_{i-1}\right) \leq \exp \left\{\lambda^{\beta} \mathbf{E}\left(\left(\xi_{i}^{-}\right)^{\beta} \mid \mathcal{F}_{i-1}\right)\right\} .
$$

Proof. It is easy to see that, for all $x \in \mathbf{R}$ and $\beta \in(1,2)$,

$$
\exp \left\{x-\left(x^{+}\right)^{\beta}\right\} \leq 1+x+\left(x^{-}\right)^{\beta} .
$$

With $x=\lambda \xi_{i}$, we easily obtain, for all $\lambda \geq 0$,

$$
\exp \left\{\lambda \xi_{i}-\left(\lambda \xi_{i}^{+}\right)^{\beta}\right\} \leq 1+\lambda \xi_{i}+\left(\lambda \xi_{i}^{-}\right)^{\beta}
$$

Taking conditional expectations on both sides of (35), we get

$$
\mathbf{E}\left(\exp \left\{\lambda \xi_{i}-\lambda^{\beta}\left(\xi_{i}^{+}\right)^{\beta}\right\} \mid \mathcal{F}_{i-1}\right) \leq 1+\lambda^{\beta} \mathbf{E}\left(\left(\xi_{i}^{-}\right)^{\beta} \mid \mathcal{F}_{i-1}\right)
$$

Using the inequality $1+x \leq e^{x}$, we obtain the desired inequality.

Proof of Theorem 2.2. For given $x, v>0$, define the stopping time $T$ :

$$
T=\min \left\{k \in[1, n]: S_{k} \geq x \text { and } \mathrm{G}_{k}^{0}(\beta) \leq v^{\beta}\right\},
$$

with the convention that $\min \emptyset=0$. Then we have

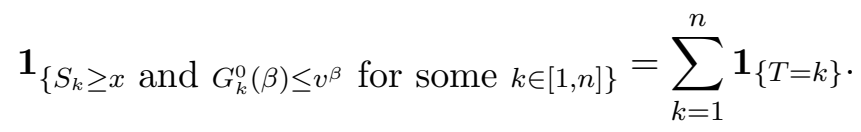

Denote by $\mathbf{E}_{\lambda}$ the expectation with respect to the conjugate probability measure (34). 
Using the change of measure (34), we get, for all $x, \lambda, v>0$,

$$
\begin{aligned}
& \mathbf{P}\left(S_{k} \geq x \text { and } \mathrm{G}_{k}^{0}(\beta) \leq v^{\beta} \text { for some } k \in[1, n]\right) \\
= & \mathbf{E}_{\lambda}\left(Z_{T \wedge n}(\lambda)^{-1} \mathbf{1}_{\left\{S_{k} \geq x\right.} \text { and } G_{k}^{0}(\beta) \leq v^{2} \text { for some } k \in[1, n]\right\} \\
= & \sum_{k=1}^{n} \mathbf{E}_{\lambda}\left(\exp \left\{-\lambda S_{k}+\lambda^{\beta} \sum_{i=1}^{k}\left(\xi_{i}^{+}\right)^{\beta}+\widehat{\Psi}_{k}(\lambda)\right\} \mathbf{1}_{\{T=k\}}\right),
\end{aligned}
$$

where

$$
\widehat{\Psi}_{k}(\lambda)=\sum_{i=1}^{k} \log \mathbf{E} \exp \left\{\lambda \xi_{i}-\left(\lambda \xi_{i}^{+}\right)^{\beta}\right\}
$$

From inequality (36), by Lemma 5.1, it follows that, for all $x, \lambda, v>0$,

$$
\begin{aligned}
& \mathbf{P}\left(S_{k} \geq x \text { and } \mathrm{G}_{k}^{0}(\beta) \leq v^{\beta} \text { for some } k \in[1, n]\right) \\
& \quad \leq \sum_{k=1}^{n} \mathbf{E}_{\lambda}\left(\exp \left\{-\lambda S_{k}+\lambda^{\beta} \mathrm{G}_{k}^{0}(\beta)\right\} \mathbf{1}_{\{T=k\}}\right) .
\end{aligned}
$$

Since $S_{k} \geq x$ and $\mathrm{G}_{k}^{0}(\beta) \leq v^{\beta}$ on the set $\{T=k\}$, we obtain, for all $x, \lambda, v>0$,

$$
\mathbf{P}\left(S_{k} \geq x \text { and } \mathrm{G}_{k}^{0}(\beta) \leq v^{\beta} \text { for some } k \in[1, n]\right) \leq \exp \left\{-\lambda x+\lambda^{\beta} v^{\beta}\right\} .
$$

The last inequality attains its minimum at

$$
\lambda=\lambda(x)=\left(\frac{x}{\beta v^{\beta}}\right)^{\frac{1}{\beta-1}} .
$$

Substituting $\lambda=\lambda(x)$ in (37), we get the desired inequality.

\section{6. $\quad$ Proof of Theorem 2.3}

Assume that $\left(\xi_{i}, \mathcal{F}_{i}\right)_{i=0, \ldots, n}$ is a sequence of martingale differences. For any nonnegative numbers $y$ and $\lambda$, define the exponential multiplicative martingale $Z(\lambda)=$ $\left(Z_{k}(\lambda), \mathcal{F}_{k}\right)_{k=0, \ldots, n}$, where

$$
Z_{k}(\lambda)=\prod_{i=1}^{k} \frac{\exp \left\{\lambda \xi_{i}-\frac{1}{2}\left(\lambda \xi_{i}\right)^{2} \mathbf{1}_{\left\{\left|\xi_{i}\right|>y\right\}}\right\}}{\mathbf{E}\left(\exp \left\{\lambda \xi_{i}-\frac{1}{2}\left(\lambda \xi_{i}\right)^{2} \mathbf{1}_{\left\{\left|\xi_{i}\right|>y\right\}}\right\} \mid \mathcal{F}_{i-1}\right)}, \quad Z_{0}(\lambda)=1
$$

If $T$ is a stopping time, then $Z_{T \wedge k}(\lambda), \lambda>0$, is also a martingale, where

$$
Z_{T \wedge k}(\lambda)=\prod_{i=1}^{T \wedge k} \frac{\exp \left\{\lambda \xi_{i}-\frac{1}{2}\left(\lambda \xi_{i}\right)^{2} \mathbf{1}_{\left\{\left|\xi_{i}\right|>y\right\}}\right\}}{\mathbf{E}\left(\exp \left\{\lambda \xi_{i}-\frac{1}{2}\left(\lambda \xi_{i}\right)^{2} \mathbf{1}_{\left\{\left|\xi_{i}\right|>y\right\}}\right\} \mid \mathcal{F}_{i-1}\right)}, \quad Z_{0}(\lambda)=1
$$


Then for any nonnegative number $\lambda$, we have the following conjugate probability measure $\mathbf{P}_{\lambda}$ on $(\Omega, \mathcal{F})$ :

$$
d \mathbf{P}_{\lambda}=Z_{T \wedge n}(\lambda) d \mathbf{P}
$$

Lemma 6.1 Assume that $\left(\xi_{i}, \mathcal{F}_{i}\right)_{i=0, \ldots, n}$ is a sequence of conditionally symmetric martingale differences. For all $\lambda, y \geq 0$, it holds

$\mathbf{E}\left(\exp \left\{\lambda \xi_{i}-\frac{1}{2}\left(\lambda \xi_{i}\right)^{2} \mathbf{1}_{\left\{\left|\xi_{i}\right|>y\right\}}\right\} \mid \mathcal{F}_{i-1}\right) \leq \exp \left\{\left(\frac{\cosh (\lambda y)-1}{y^{2}}\right) \mathbf{E}\left(\xi_{i}^{2} \mathbf{1}_{\left\{\left|\xi_{i}\right| \leq y\right\}} \mid \mathcal{F}_{i-1}\right)\right\}$,

where by convention $\frac{\cosh (\lambda y)-1}{y^{2}}=\frac{\lambda^{2}}{2}$ when $y=0$.

Proof. Let $y \geq 0$. When $\left|\xi_{i}\right| \leq y$, it follows that $\xi_{i}^{2 k} \leq y^{2 k-2} \xi_{i}^{2}$ and that

$$
\cosh \left(\lambda \xi_{i}\right) \leq 1+\frac{\xi_{i}^{2}}{y^{2}} \sum_{k=1}^{\infty} \frac{(\lambda y)^{2 k}}{(2 k) !}=1+\frac{\xi_{i}^{2}}{y^{2}}(\cosh (\lambda y)-1)
$$

When $\left|\xi_{i}\right|>y, \operatorname{since} \cosh (x) \leq \exp \left\{\frac{1}{2} x^{2}\right\}$ for all $x \in \mathbf{R}$, it follows that, for all $\lambda>0$,

$$
\left(\cosh \left(\lambda \xi_{i}\right)\right) \exp \left\{-\frac{1}{2}\left(\lambda \xi_{i}\right)^{2}\right\} \leq 1
$$

Combining (39) and (40) together, we find that, for all $\lambda, y \geq 0$,

$$
\left(\cosh \left(\lambda \xi_{i}\right)\right) \exp \left\{-\frac{1}{2}\left(\lambda \xi_{i}\right)^{2} \mathbf{1}_{\left\{\left|\xi_{i}\right|>y\right\}}\right\} \leq 1+\lambda \xi_{i}+\left(\frac{e^{\lambda y}-1-\lambda y}{y^{2}}\right) \xi_{i}^{2} \mathbf{1}_{\left\{\left|\xi_{i}\right| \leq y\right\}} .
$$

Taking conditional expectations on both sides of the last inequality, we have, for all $\lambda, y \geq 0$,

$$
\begin{aligned}
& \mathbf{E}\left(\left(\cosh \left(\lambda \xi_{i}\right)\right) \exp \{-\right.\left.\left.\frac{1}{2}\left(\lambda \xi_{i}\right)^{2} \mathbf{1}_{\left\{\left|\xi_{i}\right|>y\right\}}\right\} \mid \mathcal{F}_{i-1}\right) \\
& \leq 1+\left(\frac{\cosh (\lambda y)-1}{y^{2}}\right) \mathbf{E}\left(\xi_{i}^{2} \mathbf{1}_{\left\{\left|\xi_{i}\right| \leq y\right\}} \mid \mathcal{F}_{i-1}\right)
\end{aligned}
$$

Since $\left(\xi_{i}, \mathcal{F}_{i}\right)_{i=0, \ldots, n}$ are conditionally symmetric, it holds

$$
\begin{aligned}
\mathbf{E}\left(\left(\exp \left\{\lambda \xi_{i}\right\}\right) \exp \right. & \left.\left\{-\frac{1}{2}\left(\lambda \xi_{i}\right)^{2} \mathbf{1}_{\left\{\left|\xi_{i}\right|>y\right\}}\right\} \mid \mathcal{F}_{i-1}\right) \\
& =\mathbf{E}\left(\left(\exp \left\{-\lambda \xi_{i}\right\}\right) \exp \left\{-\frac{1}{2}\left(\lambda \xi_{i}\right)^{2} \mathbf{1}_{\left\{\left|\xi_{i}\right|>y\right\}}\right\} \mid \mathcal{F}_{i-1}\right) .
\end{aligned}
$$


Note that $\cosh \left(\lambda \xi_{i}\right)=\frac{1}{2}\left(\exp \left\{\lambda \xi_{i}\right\}+\exp \left\{-\lambda \xi_{i}\right\}\right)$. Hence (42) implies that

$$
\begin{aligned}
\mathbf{E}\left(\left(\cosh \left(\lambda \xi_{i}\right)\right) \exp \{\right. & \left.\left.-\frac{1}{2}\left(\lambda \xi_{i}\right)^{2} \mathbf{1}_{\left\{\left|\xi_{i}\right|>y\right\}}\right\} \mid \mathcal{F}_{i-1}\right) \\
& =\mathbf{E}\left(\left(\exp \left\{\lambda \xi_{i}\right\}\right) \exp \left\{-\frac{1}{2}\left(\lambda \xi_{i}\right)^{2} \mathbf{1}_{\left\{\left|\xi_{i}\right|>y\right\}}\right\} \mid \mathcal{F}_{i-1}\right) \cdot(
\end{aligned}
$$

Combining (41) and (43) together, we obtain

$\mathbf{E}\left(\exp \left\{\lambda \xi_{i}-\frac{1}{2}\left(\lambda \xi_{i}\right)^{2} \mathbf{1}_{\left\{\left|\xi_{i}\right|>y\right\}}\right\} \mid \mathcal{F}_{i-1}\right) \leq 1+\left(\frac{\cosh (\lambda y)-1}{y^{2}}\right) \mathbf{E}\left(\xi_{i}^{2} \mathbf{1}_{\left\{\left|\xi_{i}\right| \leq y\right\}} \mid \mathcal{F}_{i-1}\right)$.

Using the inequality $1+x \leq e^{x}$, we obtain the desired inequality.

Proof of Theorem 2.3. For any $y \geq 0$ and any $x, v>0$, define the stopping time $T$ :

$$
T(x, y, v)=\min \left\{k \in[1, n]: S_{k} \geq x \text { and } G_{k}^{y} \leq v^{2}\right\}
$$

with the convention that $\min \emptyset=0$. Then it follows that

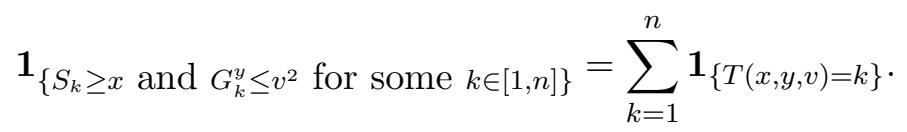

Denote $\mathbf{E}_{\lambda}$ the expectation with respect to $\mathbf{P}_{\lambda}$. Using the change of probability measure (38), we have, for all $x, \lambda, v>0$ and all $y \geq 0$,

$$
\begin{aligned}
& \mathbf{P}\left(S_{k} \geq x \text { and } G_{k}^{y} \leq v^{2} \text { for some } k \in[1, n]\right) \\
= & \left.\mathbf{E}_{\lambda}\left(Z_{T \wedge n}(\lambda)^{-1} \mathbf{1}_{\left\{S_{k} \geq x\right.} \text { and } G_{k}^{y} \leq v^{2} \text { for some } k \in[1, n]\right\}\right) \\
= & \sum_{k=1}^{n} \mathbf{E}_{\lambda}\left(\exp \left\{-\lambda S_{k}+\frac{\lambda^{2}}{2}[S]_{k}(y)+\breve{\Psi}_{k}(\lambda)\right\} \mathbf{1}_{\{T(x, y, v)=k\}}\right)
\end{aligned}
$$

where $[S]_{k}(y)=\sum_{i=1}^{k} \xi_{i}^{2} \mathbf{1}_{\left\{\left|\xi_{i}\right|>y\right\}}$ and

$$
\breve{\Psi}_{k}(\lambda)=\sum_{i=1}^{k} \log \mathbf{E}\left(\exp \left\{\lambda \xi_{i}-\frac{1}{2}\left(\lambda \xi_{i}\right)^{2} \mathbf{1}_{\left\{\left|\xi_{i}\right|>y\right\}}\right\} \mid \mathcal{F}_{i-1}\right)
$$

Since the function $f(y)=\frac{\cosh (\lambda y)-1}{y^{2}}$ is increasing in $y \geq 0$ and $f(0)=\lambda^{2} / 2$, we have

$$
\frac{\lambda^{2}}{2} \leq \frac{\cosh (\lambda y)-1}{y^{2}} \text { for all } y, \lambda>0
$$

By Lemma 4.1, we find that

$$
\widetilde{\Psi}_{k}(\lambda) \leq\left(\frac{\cosh (\lambda y)-1}{y^{2}}\right)\langle S\rangle_{k}(y)
$$


where $\langle S\rangle_{k}(y)=\sum_{i=1}^{k} \mathbf{E}\left(\xi_{i}^{2} \mathbf{1}_{\left\{\left|\xi_{i}\right|>y\right\}} \mid \mathcal{F}_{i-1}\right)$. Then, from equality (44), it follows that, for all $x, \lambda, v>0$ and all $y \geq 0$,

$$
\begin{aligned}
& \mathbf{P}\left(S_{k} \geq x \text { and } G_{k}^{y} \leq v^{2} \text { for some } k \in[1, n]\right) \\
\leq & \sum_{k=1}^{n} \mathbf{E}_{\lambda}\left(\exp \left\{-\lambda S_{k}+\frac{\lambda^{2}}{2}[S]_{k}(y)+\left(\frac{\cosh (\lambda y)-1}{y^{2}}\right)\langle S\rangle_{k}(y)\right\} \mathbf{1}_{\{T=k\}}\right) \\
\leq & \sum_{k=1}^{n} \mathbf{E}_{\lambda}\left(\exp \left\{-\lambda S_{k}+\left(\frac{\cosh (\lambda y)-1}{y^{2}}\right) G_{k}^{y}\right\} \mathbf{1}_{\{T=k\}}\right) .
\end{aligned}
$$

By the fact $S_{k} \geq x$ and $G_{k}^{y} \leq v^{2}$ on the set $\{T(x, y, v)=k\}$, inequality (45) implies that, for all $x, \lambda, v>0$ and all $y \geq 0$,

$$
\begin{aligned}
& \mathbf{P}\left(S_{k} \geq x \text { and } G_{k}^{y} \leq v^{2} \text { for some } k \in[1, n]\right) \\
\leq & \exp \left\{-\lambda x+\left(\frac{\cosh (\lambda y)-1}{y^{2}}\right) v^{2}\right\} .
\end{aligned}
$$

The last inequality attains its minimum at

$$
\lambda=\lambda(x)=\frac{1}{y} \log \left(\sqrt{1+\frac{x^{2} y^{2}}{v^{4}}}+\frac{x y}{v^{2}}\right) .
$$

Substituting $\lambda=\lambda(x)$ in (46), we obtain the desired inequality. This completes the proof of Theorem 2.3 .

\section{Acknowledgements}

We would like to thank Igal Sason for his helpful remarks and suggestions.

\section{References}

[1] Bennett, G., 1962. Probability inequalities for the sum of independent random variables. J. Amer. Statist. Assoc. 57, No. 297, 33-45.

[2] Bercu, B. and Touati, A., 2008. Exponential inequalities for self-normalized martingales with applications. Ann. Appl. Probab. 18(5): 1848-1869.

[3] Bernstein, S. N., 1927. Theorem of Probability. Moscow.

[4] De la Peña, V. H., Pang, G., 2009. Exponential inequalities for self-normalized processes with applications. Electron. Commun. Probab. 14: 372-381.

[5] De la Peña, V. H., 1999. A general class of exponential inequalities for martingales and ratios. Ann. Probab. 27, No. 1, 537-564.

[6] Delyon, B., 2009. Exponential inequaties for sums of weakly dependent variables. Electronic J. Probab. 14, No. $28,752-779$.

[7] Dzhaparidze, K. and van Zanten, J. H., 2001. On Bernstein-type inequalities for martingales. Stochastic Process. Appl. 93, 109-117.

[8] Fan, X., Grama, I. and Liu, Q., 2015. Exponential inequalities for martingales with applications. Electron. J. Probab. 20, no. 1, 1-22.

[9] Freedman, D. A., 1975. On tail probabilities for martingales. Ann. Probab. 3, No. 1, 100-118.

[10] Jing, B. Y., Liang, H. Y. and Zhou, W., 2012. Self-normalized moderate devioations for independent random variables. Sci. China Math. 55(11), 2297-2315.

[11] Raginsky, M. and Sason, I., 2013. Concentration of measure inequalities in information theory, communications, and coding. Foundations and Trends ${ }^{\circledR}$ in Communications and Information Theory 10, no. 1-2, $1-246$. 
[13] Shao, Q. M., 1997. Self-normalized large deviations. Ann. Probab. 25(1): 285-328.

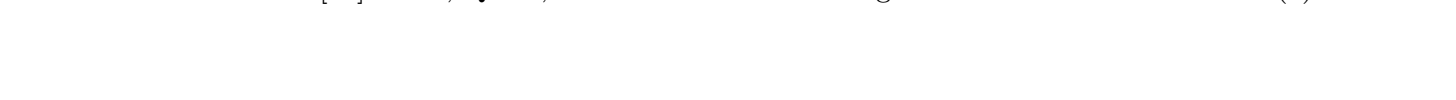

\begin{abstract}
Sason, I., 2013. Tightened exponential bounds for discrete-time conditionally symmetric martingales with
bounded jumps. Statist. Probab. Lett. 83, 1928-1936.
Sason, I., 2013. Tightened exponential bounds for discrete-time conditionally symmetric martingales with
bounded jumps. Statist. Probab. Lett. 83, 1928-1936.
\end{abstract}

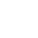

(2) 